\title{
Risk-based Stochastic Continuous-time Scheduling of Flexibility Reserve for Energy Storage Systems
}

\author{
Bosong Li, Majid Majidi, Masood Parvania \\ The University of Utah \\ \{bosong.li, majid.majidi, masood.parvania\}@utah.edu
}

\author{
Raymond Byrne \\ Sandia National Laboratories \\ rhbyrne@sandia.gov
}

\begin{abstract}
This paper develops a novel risk-based stochastic continuous-time model for optimizing the role of energy storage (ES) systems in managing the financial risk imposed to power system operation by large-scale integration of uncertain renewable energy sources (RES). The proposed model is formulated as a two-stage continuous-time stochastic optimization problem, where the generation of generating units, charging and discharging power of ES, as well as flexibility reserve capacity from both resources are scheduled in the first stage, while the flexibility reserve is deployed in the second stage to offset the uncertainty of RES generation in each scenario. The Conditional Value at Risk (CVaR) is integrated as the risk metric measuring the average of the higher tail of the system operation costs. The proposed model is implemented on the IEEE Reliability Test System using load and solar power data of CAISO. Numerical results demonstrate that the proposed model enables the system operators to effectively utilize the flexibility of ES and generating units to minimize the system operation cost and renewable energy curtailment at a given risk tolerance level.
\end{abstract}

\section{Introduction}

\subsection{Background and Literature Review}

Large-scale integration of renewable energy sources (RES) in power systems is adding to the sources of variability and uncertainty and is calling for enhancing the flexibility of power systems operation. This, in turn, is imposing additional financial burden and risk on the system operators, who would need to acquire additional flexibility resources to secure the system operation against the variability and uncertainty of RES. In essence, the system operators face a trade-off between the increasing cost of flexibility services and curtailing RES: the system operators would need to either acquire more services to enhance the operational flexibility of the system and accommodate RES, or curtail RES to ensure the operational reliability of the system.
Energy storage (ES) systems, for their ability to provide flexibility with high ramping capability, are seen as the most promising solution to provide the flexibility needed to curb the uncertainty and variability of RES in power systems [1]. In this regard, FERC Order 841 mandated the independent system operators (ISOs) and regional transmission operators (RTOs) in the U.S. to develop market rules and practices for enabling ES participation and competitiveness in their markets [2]. Multiple research works are also conducted on enabling ES systems for providing services to enhance the economic and reliable operation of power system [3-10]. In [3], a novel market framework is proposed to embrace the participation of ES in both energy and reserve markets. The compressed-air energy storage (CAES) systems are employed in the day-ahead and real-time system operation to provide services in energy, regulation, spinning reserve, and non-spinning reserve markets [4]. In [5], the flexible ramping product provided by ES is explored in the day-ahead market. The application of ES in energy and frequency regulation markets to enhance operation of MISO system is investigated in [6]. The ES charging/discharging power is utilized in [7] to minimize the system peak load level, which contributes to the utility revenue maximization through resilience improvement of the system. In [8], energy storage is utilized to facilitate the integration of renewable energy resources by providing energy-shifting and fast-ramping capability. Continuous-time look-ahead optimization models are proposed in $[9,10]$ for optimal scheduling of ES systems to provide balancing energy and regulation capacity in real-time markets.

By providing various flexibility services, ES systems can contribute to managing the financial risk associated with RES integration. In [11,12], the Conditional Value at Risk (CVaR) is employed to manage the financial risk associated with coordinated participation of ES in energy and ancillary services markets. CVaR is merged with constraints in the optimal system scheduling model in [13], where ES is utilized to hedge the risk of 
the system from multiple RES integration scenarios. A CVaR-constrained model is developed in [14] for modeling ES integration in an energy hub to manage the risk associated with the uncertainties of electricity price, demand, and solar generation. In [15], CVaR is utilized to quantify the financial loss due to wind power fluctuation beyond the regulation capacity of the system. Despite the current modeling efforts reviewed above, technical literature lacks a model for assisting power system operators to quantify and optimize the role of ES systems in managing the financial risk associated with RES integration. Such model should accurately capture the flexibility and ramping capability of ES systems in risk management strategy of systems operators

\subsection{Contribution and paper structure}

This paper proposes a risk-based stochastic continuous-time optimization model for optimal scheduling of flexibility reserve for generating units and ES systems in day-ahead power systems operation, while minimizing the system operation cost at the desired financial risk level against the uncertainty of load and RES generation. More specifically, in the proposed model, formulated as a two-stage stochastic optimization model, the power generation of generating units, the charging and discharging power of ES systems, as well as the flexibility reserve capacity from both resources are determined in the first stage, while the flexibility reserve deployment from generating units and ES systems is decided on the second stage, given the realization of uncertain load and RES generation scenarios. The proposed model integrates a linearized calculation of CVaR as the risk measure for managing the financial risk of system operation imposed by the variability and uncertainty of the load and RES. The proposed model integrates an accurate continuous-time model of ES operation constraints, which enables capturing the ultimate flexibility and value of ES systems in compensating the variability and uncertainty of RES in the system operation. The computation intractability of the continuous-time optimization problem is dealt with through a function space solution methodology, which converts the proposed model into a solvable mixed-integer linear programming (MILP). The application of the proposed model enables the system operator to make a trade-off between employing more flexibility reserve to accommodate the uncertainty, and curtailing more renewable generation to avoid the underlying uncertainty, given the desired risk level.

The rest of this paper is organized as follows: formulation of the proposed risk-based stochastic continuous-time model along with the CVaR calculation model are presented in Section 2. In Section 3, a function space solution methodology using Bernstein polynomials is proposed to convert the proposed model to a solvable MILP problem. Numerical results conducted on the IEEE-RTS using load and solar power data from CAISO are presented in Section 4, and the conclusions are drawn in Section 5.

\section{Risk-based Stochastic Continuous-time Optimization Model}

The proposed risk-based stochastic continuous-time optimization model for scheduling flexibility reserve of generating units and ES systems is formulated in this section as a two-stage stochastic optimization problem. The objective of the proposed model is to minimize the total system operation cost plus the financial risk measured by $\mathrm{CVaR}$, which is formulated as follows:

$$
\min (1-\beta) \cdot F+\beta \cdot C V a R
$$

where $F$ is the expected total operation cost, and $\beta$ is the risk aversion coefficient showing the system operator's risk preference. The more risk-averse the system operator is, the higher $\beta$ is, and vice versa.

1) Modeling Operation Cost: The total operation cost $F$ (the first term in (1)) is formulated as follows:

$$
\begin{aligned}
& F=\int_{T}\left[C^{G}(\mathbf{G}(t), \mathbf{I}(t))+\mathbf{1}^{K}(\mathbf{S U}(t)+\mathbf{S D}(t))\right. \\
& +\gamma^{G, S} \mathbf{G}^{S}(t)-\gamma^{D, S} \mathbf{D}^{S}(t) \\
& +\boldsymbol{\mu}^{u, G} \mathbf{R}^{u, G}(t)+\boldsymbol{\mu}^{d, G} \mathbf{R}^{d, G}(t) \\
& +\boldsymbol{\mu}^{u, S}\left(\mathbf{R}^{u, G^{S}}(t)+\mathbf{R}^{u, D^{S}}(t)\right) \\
& \left.+\boldsymbol{\mu}^{d, S}\left(\mathbf{R}^{d, G^{S}}(t)+\mathbf{R}^{d, D^{S}}(t)\right)\right] d t \\
& +\int_{T} \int_{\Omega}\left(\boldsymbol{\rho}^{u, G} \mathbf{r}_{\omega}^{u, G}(t)+\boldsymbol{\rho}^{d, G} \mathbf{r}_{\omega}^{d, G}(t)\right) \mathbb{P}(\omega) d \omega d t \\
& +\int_{T} \int_{\Omega}\left(\boldsymbol{\rho}^{u, G^{S}} \mathbf{r}_{\omega}^{u, G^{S}}(t)+\boldsymbol{\rho}^{d, G^{S}} \mathbf{r}_{\omega}^{d, G^{S}}(t)\right) \mathbb{P}(\omega) d \omega d t \\
& +\int_{T} \int_{\Omega}\left(\boldsymbol{\rho}^{u, D^{S}} \mathbf{r}_{\omega}^{u, D^{S}}(t)+\boldsymbol{\rho}^{d, D^{S}} \mathbf{r}_{\omega}^{d, D^{S}}(t)\right) \mathbb{P}(\omega) d \omega d t \\
& +\int_{T} \int_{\Omega}\left(\boldsymbol{v}^{u, G} \dot{\mathbf{r}}_{\omega}^{G, u p}(t)+\boldsymbol{v}^{d, G} \dot{\mathbf{r}}_{\omega}^{G, d n}(t)\right) \mathbb{P}(\omega) d \omega d t \\
& +\int_{T} \int_{\Omega}\left(\boldsymbol{v}^{u, S} \dot{\mathbf{r}}_{\omega}^{S, u p}(t)+\boldsymbol{v}^{d, S} \dot{\mathbf{r}}_{\omega}^{S, d n}(t)\right) \mathbb{P}(\omega) d \omega d t \\
& +\int_{T} \int_{\Omega} \mu^{S P} S P_{\omega}(t) \mathbb{P}(\omega) d \omega d t
\end{aligned}
$$


The total operation cost $F$ in (2) consists of the first-stage and second-stage costs. The first-stage cost includes the first integral of $F$ in (2), which consist of the total generation cost, start-up and shut-down costs of generating units, discharging cost and charging utility of ES system and up and down flexibility reserve capacity costs of generating units and ES system. Line 1 of (2) represents the generation and start-up and shut-down costs of generating units, where $C^{G}(\mathbf{G}(t), \mathbf{I}(t))$ is the total generation cost of units, $\mathbf{G}(t)$ is day-ahead schedule of units, $\mathbf{I}(t)$ is the commitment status of units and $\mathbf{S U}(t)$ and $\mathbf{S D}(t)$ are respectively the start-up and shut-down costs of units. The total generation cost of units is formulated as follows, where the cost function of each generating unit $k$ is modeled as a piece-wise linear function with $m$ sections, and $\gamma_{k, m}(t)$ and $\Gamma_{k, m}(t)$ are the marginal cost and the corresponding power generation trajectory in each linear section:

$$
\begin{aligned}
C^{G}(\mathbf{G}(t), \mathbf{I}(t)) & =\sum_{k=1}^{K}\left(C_{k}^{G}\left(\underline{G}_{k}\right) I_{k}(t)\right. \\
& \left.+\sum_{n=0}^{M_{k}-1} \gamma_{k, m}^{G}(t) \Gamma_{k, m}(t)\right) .
\end{aligned}
$$

Line 2 of (2) represents the discharging cost and charging utility of ES system, where $\mathbf{G}^{S}(t)$ and $\mathbf{D}^{S}(t)$ are respectively the discharging and charging power trajectory, and $\gamma^{G, S}$ and $\gamma^{D, S}$ are the corresponding unit discharging cost and charging utility. Lines 3-5 of (2) formulate the flexibility reserve capacity cost of generating units and ES systems, where row vectors $\boldsymbol{\mu}^{u, G}, \boldsymbol{\mu}^{d, G}$ and $\boldsymbol{\mu}^{u, S}, \boldsymbol{\mu}^{d, S}$ are respectively up and down flexibility reserve capacity costs of generating units and ES systems. The up and down flexibility reserve capacity is denoted respectively by $\mathbf{R}^{u, G}(t)$ and $\mathbf{R}^{d, G}(t)$ for generating units, and by $\mathbf{R}^{u, G^{S}}(t)$ and $\mathbf{R}^{d, G^{S}}(t)$ for the ES system during discharging, and $\mathbf{R}^{u, D^{S}}(t)$ and $\mathbf{R}^{d, D^{S}}(t)$ for ES during charging.

The second-stage costs in (2) consist of the integral costs in lines 6-11 of (2), which formulate the flexibility reserve deployment cost, flexible ramping cost, and renewable energy spillage cost over the probability space $\Omega$, where the probability of scenario $\omega \in \Omega$ is denoted by $\mathbb{P}(\omega)$. In scenario $\omega, \mathbf{r}_{\omega}^{u, G}(t)$ and $\mathbf{r}_{\omega}^{d, G}(t)$ respectively denote the up and down flexibility reserve deployment trajectories of generating units. The up and down flexibility reserve deployment trajectories of ES systems are respectively denoted by $\mathbf{r}_{\omega}^{u, G^{S}}(t)$ and $\mathbf{r}_{\omega}^{d, G^{S}}(t)$ when discharging, and $\mathbf{r}_{\omega}^{u, D^{S}}(t)$ and $\mathbf{r}_{\omega}^{d, D^{S}}(t)$ when charging. The corresponding up and down flexible ramping trajectories are shown by $\dot{\mathbf{r}}_{\omega}^{G, u p}(t)$ and $\dot{\mathbf{r}}_{\omega}^{G, d n}(t)$ for generating units, and by $\dot{\mathbf{r}}_{\omega}^{S, u p}(t)$ and $\dot{\mathbf{r}}_{\omega}^{S, d n}(t)$ for ES systems. The up and down flexibility reserve deployment costs are respectively denoted by $\boldsymbol{\rho}^{u, G}$ and $\boldsymbol{\rho}^{d, G}$ for generating units, $\boldsymbol{\rho}^{u, G^{S}}$ and $\boldsymbol{\rho}^{d, G^{S}}$ for ES discharging, as well as $\boldsymbol{\rho}^{u, D^{S}}$ and $\boldsymbol{\rho}^{d, D^{S}}$ for ES charging. The up and down flexible ramping cost coefficients are shown by $\boldsymbol{v}^{u, G}$ and $\boldsymbol{v}^{d, G}$ for generating units, while $\boldsymbol{v}^{u, S}$ and $\boldsymbol{v}^{d, S}$ for ES systems. The spillage cost of renewable energy is denoted by renewable energy spillage cost $\mu^{S P}$ in the last line in (2), where $S P_{\omega}(t)$ is the renewable energy spillage trajectory.

2) Financial Risk Modeling using CVaR: In (1), $\mathrm{CVaR}$ is utilized to measure the financial risk imposed on power system operators by the uncertainty of renewable energy resources and load in the system operation. CVaR is a financial risk measurement approach that is extensively used in many applications, and offers several features, including monotonicity, invariance under translations, positive homogeneity, and subadditivity and convexity [16]. Under $\alpha$ confidence level, CVaR is defined as the average of the higher $(1-$ $\alpha$ ) tail of the costs in the realized uncertain scenarios, which is mathematically formulated as follows:

$$
\begin{aligned}
& C V a R=\xi+(1-\alpha)^{-1} \int_{\Omega} z_{\omega} \mathbb{P}(\omega) d \omega, \\
& z_{\omega} \geq f_{\omega}-\xi \\
& z_{\omega} \geq 0,
\end{aligned}
$$

where $\xi$ is the value at risk (VaR) that is the $(1-\alpha)$ percentile of the scenario cost under ascending order, $z_{\omega}$ is a positive auxiliary variable representing the difference between VaR and $f_{\omega}$, and $f_{\omega}$ is the operation cost in scenario $\omega$, formulated as follows:

$$
\begin{aligned}
f_{\omega}=\int_{T} & {\left[C^{G}(\mathbf{G}(t), \mathbf{I}(t))+\mathbf{1}^{K}(\mathbf{S} \mathbf{U}(t)+\mathbf{S D}(t))\right.} \\
& +\boldsymbol{\gamma}^{G, S} \mathbf{G}^{S}(t)-\boldsymbol{\gamma}^{D, S} \mathbf{D} \boldsymbol{\gamma}^{S}(t) \\
& +\boldsymbol{\mu}^{u, G} \mathbf{R}^{u, G}(t)+\boldsymbol{\mu}^{d, G} \mathbf{R}^{d, G}(t) \\
& +\boldsymbol{\mu}^{u, S}\left(\mathbf{R}^{u, G^{S}}(t)+\mathbf{R}^{u, D^{S}}(t)\right) \\
& +\boldsymbol{\mu}^{d, S}\left(\mathbf{R}^{d, G^{S}}(t)+\mathbf{R}^{d, D^{S}}(t)\right) \\
& +\boldsymbol{\rho}^{u, G} \mathbf{r}_{\omega}^{u, G}(t)+\boldsymbol{\rho}^{d, G} \mathbf{r}_{\omega}^{d, G}(t) \\
& +\boldsymbol{\rho}^{u, G^{S}} \mathbf{r}_{\omega}^{u, G^{S}}(t)+\boldsymbol{\rho}^{d, G^{S}} \mathbf{r}_{\omega}^{d, G^{S}}(t) \\
& +\boldsymbol{\rho}^{u, D^{S}} \mathbf{r}_{\omega}^{u, D^{S}}(t)+\boldsymbol{\rho}^{d, D^{S}} \mathbf{r}_{\omega}^{d, D^{S}}(t)
\end{aligned}
$$




$$
\begin{aligned}
& +\boldsymbol{v}^{u, G} \dot{\mathbf{r}}_{\omega}^{G, u p}(t)+\boldsymbol{v}^{d, G} \dot{\mathbf{r}}_{\omega}^{G, d n}(t) \\
& +\boldsymbol{v}^{u, S} \dot{\mathbf{r}}_{\omega}^{S, u p}(t)+\boldsymbol{v}^{d, S} \dot{\mathbf{r}}_{\omega}^{S, d n}(t) \\
& \left.+\mu^{S P} S P_{\omega}(t)\right] d t
\end{aligned}
$$

where lines $1-5$ of (7) are the first-stage costs and lines 6-11 are the second-stage costs associated with operating the system in scenario $\omega$. The VaR and $\mathrm{CVaR}$ as functions of the scenario operation cost $f_{\omega}$ are illustrated in Fig. 1.
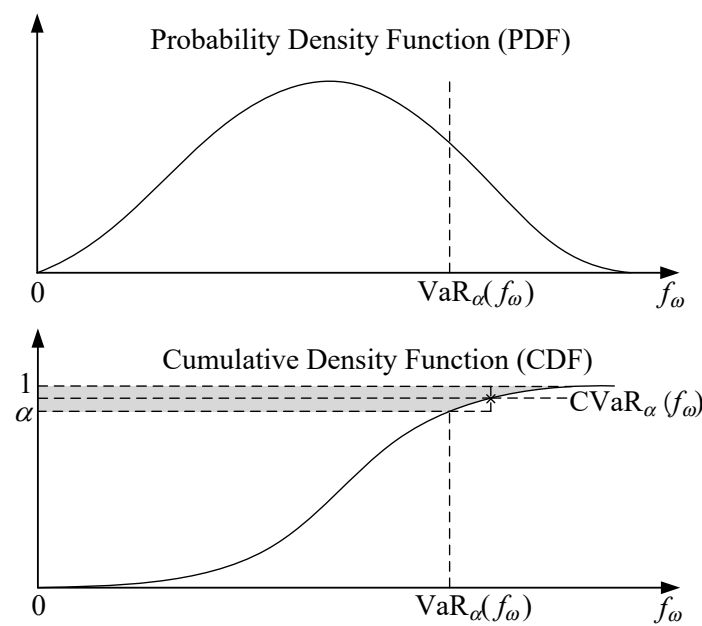

Figure 1. Illustration of Var and CVaR

3) First-stage constraints: The first-stage decisions of the proposed model include the day-ahead commitment and schedule of generating units, the charging/discharging power of ES systems, as well as flexibility reserve capacity of both resources. The first-stage constraints are formulated as follows:

$$
\begin{aligned}
& \mathbf{1}^{K} \mathbf{G}(t)+\mathbf{1}^{J}\left(\mathbf{G}^{S}(t)-\mathbf{D}^{S}(t)\right)=L_{0}(t), \\
& L_{0}(t)=D_{0}(t)-G_{0}^{R}(t), \\
& \mathbf{G}(t)+\mathbf{R}^{u, G}(t) \leq \overline{\mathbf{G}} \mathbf{I}(t), \\
& \mathbf{G}(t)-\mathbf{R}^{d, G}(t) \geq \underline{\mathbf{G}} \mathbf{I}(t), \\
& \dot{\mathbf{G}} \mathbf{I}(t)+\overline{\mathbf{G}}^{S D} \int_{t-\epsilon}^{t+\epsilon} \dot{\mathbf{I}}\left(t^{\prime}\right) d t^{\prime} \leq \dot{\mathbf{G}}(t) \\
& \dot{\mathbf{G}}(t) \leq \dot{\mathbf{G}} \mathbf{I}(t)+\dot{\mathbf{G}}^{S U} \int_{t-\epsilon}^{t+\epsilon} \dot{\mathbf{I}}\left(t^{\prime}\right) d t^{\prime}, \\
& \mathbf{S U}(t) \geq \mathbf{V} \int_{t-\epsilon}^{t+\epsilon} \dot{\mathbf{I}}\left(t^{\prime}\right) d t^{\prime}, \quad \mathbf{S U}(t) \geq 0, \\
& \mathbf{S D}(t) \geq \mathbf{W} \int_{t-\epsilon}^{t+\epsilon}-\dot{\mathbf{I}}\left(t^{\prime}\right) d t^{\prime}, \quad \mathbf{S D}(t) \geq 0,
\end{aligned}
$$

$$
\begin{aligned}
& \int_{t}^{t+\mathrm{UT}} \mathbf{I}\left(t^{\prime}\right) d t^{\prime} \geq \operatorname{Diag}(\mathbf{U T}) \int_{t-\epsilon}^{t+\epsilon} \dot{\mathbf{I}}\left(t^{\prime}\right) d t^{\prime}, \\
& \int_{t}^{t+\mathrm{DT}}\left(\mathbf{1}^{K}-\mathbf{I}\left(t^{\prime}\right)\right) d t^{\prime} \geq \operatorname{Diag}(\mathbf{D} \mathbf{T}) \int_{t-\epsilon}^{t+\epsilon}-\dot{\mathbf{I}}\left(t^{\prime}\right) d t^{\prime}, \\
& \frac{d \mathbf{E}^{S}(t)}{d t}=\boldsymbol{\eta}^{\boldsymbol{c}} \mathbf{D}^{S}(t)-\boldsymbol{\eta}^{\boldsymbol{d}^{-1}} \mathbf{G}^{S}(t), \\
& \frac{d \overline{\mathbf{E}}^{S}(t)}{d t}=\frac{d \mathbf{E}^{S}(t)}{d t}+\boldsymbol{\eta}^{c} \mathbf{R}^{d, D^{S}}(t)+\boldsymbol{\eta}^{\boldsymbol{d}^{-1}} \mathbf{R}^{d, G^{S}}(t), \\
& \frac{d \underline{\mathbf{E}}^{S}(t)}{d t}=\frac{d \mathbf{E}^{S}(t)}{d t}-\boldsymbol{\eta}^{c} \mathbf{R}^{u, D^{S}}(t)-\boldsymbol{\eta}^{\boldsymbol{d}^{-1}} \mathbf{R}^{u, G^{S}}(t), \\
& \mathbf{G}^{S}(t)+\mathbf{R}^{u, G^{S}}(t) \leq \overline{\mathbf{G}}^{S} \mathbf{I}^{S}(t), \\
& \mathbf{G}^{S}(t)-\mathbf{R}^{d, G^{S}}(t) \geq \mathbf{0}, \\
& \mathbf{D}^{S}(t)+\mathbf{R}^{d, D^{S}}(t) \leq \overline{\mathbf{D}}^{S}\left(\mathbf{1}-\mathbf{I}^{S}(t)\right), \\
& \mathbf{D}^{S}(t)-\mathbf{R}^{u, D^{S}}(t) \geq \mathbf{0}, \\
& \dot{\mathbf{G}}^{S} \leq \dot{\mathbf{G}}^{S}(t) \leq \overline{\dot{\mathbf{G}}} \text {, } \\
& \underline{\dot{\mathbf{D}}}^{S} \leq \dot{\mathbf{D}}^{S}(t) \leq \overline{\mathbf{D}} \text {, } \\
& \underline{\mathbf{E}} \leq \underline{\mathbf{E}}^{S}(t), \quad \overline{\mathbf{E}}^{S}(t) \leq \overline{\mathbf{E}}, \\
& \mathbf{I}(t), \mathbf{I}^{S}(t) \in\{0,1\},
\end{aligned}
$$

In (8), the forecast net-load mean $L_{0}(t)$ is balanced through power generation of generating units $\mathbf{G}(t)$, ES discharging power $\mathbf{G}^{S}(t)$, and ES charging power $\mathbf{D}^{S}(t)$, where $\mathbf{1}^{K}$ and $\mathbf{1}^{J}$ are respectively $K$ - and $J$ dimensional vectors consisting of one. Forecast net-load mean $L_{0}(t)$ is equal to the difference between forecast load $D_{0}(t)$ and forecast renewable energy generation $G_{0}^{R}(t)$ in (9). The power and ramping constraints of generating units are formulated in (10)-(13), where

$\underline{\mathbf{G}}, \overline{\mathbf{G}}, \underline{\mathbf{G}}$, and $\overline{\dot{\mathbf{G}}}$ are respectively the minimum and maximum power and ramping capacity of generating units. The start-up and shut-down costs are calculated respectively in (14) and (15), where $\mathbf{V}$ and $\mathbf{W}$ are diagonal matrices of start-up and shut-down cost parameters. The minimum on and off time constraints are formulated in (16) and (17), where UT and DT are minimum on and off time parameter vectors.

The state equations governing the dynamics of ES energy trajectory $\mathbf{E}^{S}(t)$, upper energy trajectory $\overline{\mathbf{E}}^{S}(t)$ considering only down flexibility reserve capacity, and lower energy trajectory $\underline{\mathbf{E}}^{S}(t)$ considering only up flexibility reserve capacity are respectively formulated in (18)-(20), where $\eta^{c}$ and $\eta^{d}$ are respectively charging and discharging efficiencies of ES system. The 
discharging and charging power, ramping, and energy constraints of ES systems are respectively imposed in (21)-(27), where $\overline{\mathbf{G}}^{S}$ and $\overline{\mathbf{D}}^{S}$ are discharging and charging power rates of ES systems, and $\underline{\mathbf{E}}$ and $\overline{\mathbf{E}}$ are the minimum and maximum ES energy capacity; the minimum and maximum ramping limitations are respectively denoted by $\dot{\dot{\mathbf{G}}}^{S}$ and $\overline{\mathbf{G}}^{S}$ for ES discharging, and by $\underline{\mathbf{D}}^{S}$ and $\overline{\mathbf{D}}^{S}$ for ES charging. The commitment status of generating units and charging/discharging status of ES systems are denoted by binary variables $\mathbf{I}(t)$ and $\mathbf{I}^{S}(t)$ in (28).

4) Second-stage constraints: the second-stage decisions include the flexibility reserve deployment and RES curtailment decisions in each scenario $\omega$. The second-stage constraints are formulated as follows:

$$
\begin{aligned}
& \mathbf{1}^{K}\left(\mathbf{G}(t)+\mathbf{r}_{\omega}^{u, G}(t)-\mathbf{r}_{\omega}^{d, G}(t)\right) \\
& \mathbf{1}^{J}\left(\mathbf{G}^{S}(t)+\mathbf{r}_{\omega}^{u, G^{S}}(t)-\mathbf{r}_{\omega}^{d, G^{S}}(t)\right) \\
& -\mathbf{1}^{J}\left(\mathbf{D}^{S}(t)+\mathbf{r}_{\omega}^{d, D^{S}}(t)-\mathbf{r}_{\omega}^{u, D^{S}}(t)\right)=L_{\omega}(t) \\
& L_{\omega}(t)=d_{\omega}(t)-\left(g_{\omega}^{R}(t)-S P_{\omega}(t)\right), \\
& -\mathbf{R}^{d, G}(t) \leq \mathbf{r}_{\omega}^{u, G}(t)-\mathbf{r}_{\omega}^{d, G}(t) \leq \mathbf{R}^{u, G}(t), \\
& -\mathbf{R}^{d, G^{S}}(t) \leq \mathbf{r}_{\omega}^{u, G^{S}}(t)-\mathbf{r}_{\omega}^{d, G^{S}}(t) \leq \mathbf{R}^{u, G^{S}}(t), \\
& -\mathbf{R}^{d, D^{S}}(t) \leq \mathbf{r}_{\omega}^{u, D^{S}}(t)-\mathbf{r}_{\omega}^{d, D^{S}}(t) \leq \mathbf{R}^{u, D^{S}}(t),(33) \\
& \dot{\mathbf{G}}(t)+\left(\dot{\mathbf{r}}_{\omega}^{u, G}(t)-\dot{\mathbf{r}}_{\omega}^{d, G}(t)\right) \geq \underline{\mathbf{G}} \mathbf{I}(t)+\overline{\mathbf{G}}^{S D} \int_{t-\epsilon}^{t+\epsilon} \dot{\mathbf{I}}\left(t^{\prime}\right) d t^{\prime},
\end{aligned}
$$$$
\dot{\mathbf{G}}(t)+\left(\dot{\mathbf{r}}_{\omega}^{u, G}(t)-\dot{\mathbf{r}}_{\omega}^{d, G}(t)\right) \leq \dot{\mathbf{G}} \mathbf{I}(t)+\dot{\overline{\mathbf{G}}}^{S U} \int_{t-\epsilon}^{t+\epsilon} \dot{\mathbf{I}}\left(t^{\prime}\right) d t^{\prime},
$$

$\dot{\mathbf{G}}^{S} \leq \dot{\mathbf{G}}^{S}(t)+\dot{\mathbf{r}}_{\omega}^{u, G^{S}}(t)-\dot{\mathbf{r}}_{\omega}^{d, G^{S}}(t) \leq \overline{\mathbf{G}}^{S}$,

$\dot{\mathbf{D}}^{S} \leq \dot{\mathbf{D}}^{S}(t)-\dot{\mathbf{r}}_{\omega}^{u, D^{S}}(t)+\dot{\mathbf{r}}_{\omega}^{d, D^{S}}(t) \leq \overline{\mathbf{D}}^{S}$,

$\dot{\mathbf{r}}_{\omega}^{G}(t)=\dot{\mathbf{r}}_{\omega}^{u, G}(t)-\dot{\mathbf{r}}_{\omega}^{d, G}(t)$,

$\dot{\mathbf{r}}_{\omega}^{G^{S}}(t)=\dot{\mathbf{r}}_{\omega}^{u, G^{S}}(t)-\dot{\mathbf{r}}_{\omega}^{d, G^{S}}(t)$,

$\dot{\mathbf{r}}_{\omega}^{D^{S}}(t)=\dot{\mathbf{r}}_{\omega}^{d, D^{S}}(t)-\dot{\mathbf{r}}_{\omega}^{u, D^{S}}(t)$,

$\dot{\mathbf{r}}_{\omega}^{G, u p}(t)-\dot{\mathbf{r}}_{\omega}^{G, d n}(t)=\dot{\mathbf{r}}_{\omega}^{G}(t)$,

$\dot{\mathbf{r}}_{\omega}^{S, u p}(t)-\dot{\mathbf{r}}_{\omega}^{S, d n}(t)=\dot{\mathbf{r}}_{\omega}^{G^{S}}(t)-\dot{\mathbf{r}}_{\omega}^{D^{S}}(t)$,

$\frac{d \mathbf{E}_{\omega}^{S}(t)}{d t}=\frac{d \mathbf{E}^{S}(t)}{d t}+\boldsymbol{\eta}^{c}\left(\mathbf{r}_{\omega}^{d, D^{S}}(t)-\mathbf{r}_{\omega}^{u, D^{S}}(t)\right)$

$$
-\boldsymbol{\eta}^{\boldsymbol{d}^{-1}}\left(\mathbf{r}_{\omega}^{u, G^{S}}(t)-\mathbf{r}_{\omega}^{d, G^{S}}(t)\right),
$$

$\underline{\mathbf{E}} \leq \mathbf{E}_{\omega}^{S}(t) \leq \overline{\mathbf{E}}$,

$\mathbf{0} \leq \mathbf{S P}_{\omega}(t) \leq \mathbf{g}_{\omega}^{R}(t)$,

$\dot{\mathbf{r}}_{\omega}^{G, u p}(t), \dot{\mathbf{r}}_{\omega}^{G, d n}(t), \dot{\mathbf{r}}_{\omega}^{S, u p}(t), \dot{\mathbf{r}}_{\omega}^{S, d n}(t) \geq 0$.

The second-stage power balance constraint is formulated in (29), where the first-stage schedule of generating units and ES systems is adjusted by flexibility reserve deployment variables $\mathbf{r}_{\omega}^{u, G}(t)$ and $\mathbf{r}_{\omega}^{d, G}(t)$ for generating units, as well as $\mathbf{r}_{\omega}^{u, G^{S}}(t)$, $\mathbf{r}_{\omega}^{d, G^{S}}(t), \mathbf{r}_{\omega}^{u, D^{S}}(t)$, and $\mathbf{r}_{\omega}^{d, D^{S}}(t)$ for ES systems, in order to supply the net-load realization in scenario $\omega$, shown by $L_{\omega}(t)$. The net-load realization $L_{\omega}(t)$ is equal to the load $d_{\omega}(t)$, minus renewable energy generation $g_{\omega}^{R}(t)$, plus renewable energy spillage $S P_{\omega}(t)$ in each realized scenario, as formulated in (30).

The flexibility reserve deployment is constrained in (31)-(33) to the flexibility reserved capacity of generating units and ES systems scheduled in the first-stage. The ramping constraints for generating units and ES systems considering flexibility reserve deployment are formulated in (34)-(37), where $\dot{\mathbf{r}}_{\omega}^{u, G}(t)$ and $\dot{\mathbf{r}}_{\omega}^{d, G}(t)$ are ramping trajectories corresponding to flexibility reserve deployment of generating units, while $\dot{\mathbf{r}}_{\omega}^{u, G^{S}}(t), \dot{\mathbf{r}}_{\omega}^{d, G^{S}}(t), \dot{\mathbf{r}}_{\omega}^{u, D^{S}}(t)$, and $\dot{\mathbf{r}}_{\omega}^{d, D^{S}}(t)$ are ramping trajectories corresponding to flexibility reserve deployment of ES systems. Auxiliary variables $\dot{\mathbf{r}}_{\omega}^{G}(t)$, $\dot{\mathbf{r}}_{\omega}^{G^{S}}(t)$, and $\dot{\mathbf{r}}_{\omega}^{D^{S}}(t)$ in (38)-(40) denote the total flexible ramping trajectories for generating units and ES systems. Positive and negative components of the total ramping trajectories are separated in (41)-(42) for each resource using non-negative auxiliary trajectories denoted with $\dot{\mathbf{r}}_{\omega}^{G, u p}(t), \dot{\mathbf{r}}_{\omega}^{G, d n}(t), \dot{\mathbf{r}}_{\omega}^{S, u p}(t)$, and $\dot{\mathbf{r}}_{\omega}^{S, d n}(t)$ in (46). The second-stage state equation of ES systems governing the dynamics of energy trajectory in scenario $\omega$, shown by $E_{\omega}^{S}(t)$, is formulated in (43), which is subject to the minimum and maximum energy capacity boundaries in (44). The RES spillage in scenario $\omega$ is constrained in (45) to the renewable generation realized in the scenario.

In summary, the proposed model is formulated as follows, which is solved in the next section:

$\min$

s.t.

\section{Solution Methodology}

The proposed stochastic continuous-time optimization model is an infinite-dimensional 
optimization problem, which is computationally intractable. We propose to reduce the dimensionality of the problem decision space by projecting the parameters and decision trajectories onto a finite-dimensional function space spanned by Bernstein polynomials defined as follows $[17,18]$ :

$$
b_{q, Q}(t)=\left(\begin{array}{l}
Q \\
q
\end{array}\right) t^{q}(1-t)^{(Q-q)}, t \in[0,1],
$$

for $q=0, \ldots, Q$. In each time interval $h \in H$ of scheduling horizon $\mathcal{T}$, where $h=0, \ldots, H-$ 1 , we define the basis function vector $\mathbf{e}^{(Q)}(t)=$ $\left(e_{1}^{(Q)}(t), \ldots, e_{P}^{(Q)}(t)\right)^{T}$ with $P=(Q+1) N$ functions, that spans $\mathcal{T}$ as follows:

$$
e_{n(Q+1)+q+1}^{(Q)}(t)=b_{q, Q}\left(\frac{t-t_{h}}{T_{h}}\right), t \in\left[t_{h}, t_{h+1}\right]
$$

where the length of each time interval in $\mathcal{T}$ is the same, denoted as $\Delta T=t_{h+1}-t_{h}$. To impose $C^{1}$ continuity of the decision trajectories, a reduced-order Bernstein function space $\mathbf{w}^{(Q)}(t)=\mathbf{B e}^{(Q)}(t)$ is employed, with the dimension of $Z=(Q-1) H+2$, where $\mathbf{B}$ is the mapping matrix. The reduced-order function space $\mathbf{w}^{(Q)}(t)$ is utilized in the following to reduce the dimensionality of trajectories in the proposed model.

1) First-stage decision trajectories: The first-stage power trajectories as well as flexibility reserve capacity trajectories are modeled in the function space spanned by $\mathbf{w}^{(Q)}(t)$ as follows:

$$
\begin{aligned}
& \mathbf{G}(t)=\mathbf{G} \mathbf{w}^{(Q)}(t), \\
& \mathbf{G}^{\mathbf{S}}(t)=\mathbf{G}^{\mathbf{S}} \mathbf{w}^{(Q)}(t), \mathbf{D}^{\mathbf{S}}(t)=\mathbf{D}^{\mathbf{S}} \mathbf{w}^{(Q)}(t), \\
& \mathbf{R}^{u, G}(t)=\mathbf{R}^{u, G} \mathbf{w}^{(Q)}(t), \mathbf{R}^{d, G}(t)=\mathbf{R}^{d, G} \mathbf{w}^{(Q)}(t), \\
& \mathbf{R}^{u, G^{S}}(t)=\mathbf{R}^{u, G^{S}} \mathbf{w}^{(Q)}(t), \mathbf{R}^{d, G^{S}}(t)=\mathbf{R}^{d, G^{S}} \mathbf{w}^{(Q)}(t), \\
& \mathbf{R}^{u, D^{S}}(t)=\mathbf{R}^{u, D^{S}} \mathbf{w}^{(Q)}(t), \mathbf{R}^{d, D^{S}}(t)=\mathbf{R}^{d, D^{S}} \mathbf{w}^{(Q)}(t),
\end{aligned}
$$

where $\mathbf{G}, \mathbf{R}^{u, G}$, and $\mathbf{R}^{d, G}$ are $K \times Z$ dimensional coefficient matrices, $\mathbf{G}^{\mathbf{S}}, \mathbf{D}^{\mathbf{S}}, \mathbf{R}^{u, G^{S}}, \mathbf{R}^{d, G^{S}}, \mathbf{R}^{u, D^{S}}$, and $\mathbf{R}^{d, D^{S}}$ are $J \times Z$ dimensional matrices for $K$ generating units and $J$ ES systems. The corresponding first-stage ramping trajectories are modeled as follows:

$$
\begin{aligned}
\dot{\mathbf{G}}(t) & =\mathbf{G} \mathcal{M} \mathbf{w}^{(Q-1)}(t)=\dot{\mathbf{G}} \mathbf{w}^{(Q-1)}(t), \\
\dot{\mathbf{G}} \mathbf{S}(t) & =\mathbf{G}^{\mathbf{S}} \mathcal{M} \mathbf{w}^{(Q-1)}(t)=\dot{\mathbf{G}^{\mathbf{S}}} \mathbf{w}^{(Q-1)}(t), \\
\dot{\mathbf{D}} \mathbf{S}(t) & =\mathbf{D}^{\mathbf{S}} \mathbf{M} \mathbf{w}^{(Q-1)}(t)=\dot{\mathbf{D}^{\mathbf{S}}} \mathbf{w}^{(Q-1)}(t),
\end{aligned}
$$

where $\mathcal{M}$ is a $Z \times(Z-H)$ matrix mapping Bernstein polynomials of degree $Q$ and $Q-1$, whereas coefficient matrix $\dot{\mathbf{G}}$ is $K \times(Z-H)$ dimensional, while $\dot{\mathbf{G}}^{S}$ and $\dot{\mathbf{D}}^{S}$ are $J \times(Z-H)$ dimensional. The first-stage ES energy trajectories are modeled as follows:

$$
\begin{aligned}
& \mathbf{E}^{S}(t)=\mathbf{E}^{S} \mathbf{w}^{(Q+1)}(t), \\
& \overline{\mathbf{E}}^{S}=\mathbf{E}^{S}+\left(\boldsymbol{\eta}^{c} \mathbf{R}^{d, D^{S}}+\boldsymbol{\eta}^{\boldsymbol{d}^{-1}} \mathbf{R}^{d, G^{S}}\right) \mathcal{N}, \\
& \underline{\mathbf{E}}^{S}=\mathbf{E}^{S}-\left(\boldsymbol{\eta}^{\boldsymbol{c}} \mathbf{R}^{u, D^{S}}+\boldsymbol{\eta}^{\boldsymbol{d}^{-1}} \mathbf{R}^{u, G^{S}}\right) \mathcal{N},
\end{aligned}
$$

where $\mathcal{N}$ is a $Z \times(Z+H)$ matrix mapping Bernstein polynomials of degree $Q$ and $Q+1$, while the energy trajectories are denoted through $J \times(Z+H)$ dimensional coefficient matrices $\mathbf{E}^{S}, \overline{\mathbf{E}}^{S}$, and $\underline{\mathbf{E}}^{S}$.

2) Second-stage decision trajectories: The second-stage flexibility reserve deployment trajectories are modeled in the function space spanned by $\mathbf{w}^{(Q)}(t)$ as follows:

$$
\begin{aligned}
& \mathbf{r}_{\omega}^{u, G}(t)=\mathbf{r}_{\omega}^{u, G} \mathbf{w}^{(Q)}(t), \mathbf{r}_{\omega}^{d, G}(t)=\mathbf{r}_{\omega}^{d, G} \mathbf{w}^{(Q)}(t), \\
& \mathbf{r}_{\omega}^{u, G^{S}}(t)=\mathbf{r}_{\omega}^{u, G^{S}} \mathbf{w}^{(Q)}(t), \mathbf{r}_{\omega}^{d, G^{S}}(t)=\mathbf{r}_{\omega}^{d, G^{S}} \mathbf{w}^{(Q)}(t), \\
& \mathbf{r}_{\omega}^{u, D^{S}}(t)=\mathbf{r}_{\omega}^{u, D^{S}} \mathbf{w}^{(Q)}(t), \mathbf{r}_{\omega}^{d, D^{S}}(t)=\mathbf{r}_{\omega}^{d, D^{S}} \mathbf{w}^{(Q)}(t),
\end{aligned}
$$

where the flexibility deployment trajectories of generating units are denoted by $K \times Z$ dimensional coefficient matrices $\mathbf{r}_{\omega}^{u, G}$ and $\mathbf{r}_{\omega}^{d, G}$, while the coefficient matrices of the ES system $\mathbf{r}_{\omega}^{u, G^{S}}, \mathbf{r}_{\omega}^{d, G^{S}}, \mathbf{r}_{\omega}^{u, D^{S}}$, and $\mathbf{r}_{\omega}^{d, D^{S}}$ are $J \times Z$ dimensional. The corresponding up and down flexible ramping trajectories are denoted by $K \times(Z-H)$ dimensional coefficient matrices $\dot{\mathbf{r}}_{\omega}^{G, u p}$ and $\dot{\mathbf{r}}_{\omega}^{G, d n}$ for generating units, while using $J \times(Z-H)$ dimensional coefficient matrices $\dot{\mathbf{r}}_{\omega}^{S, u p}$ and $\dot{\mathbf{r}}_{\omega}^{S, d n}$ for ES systems as follows:

$$
\begin{aligned}
& \dot{\mathbf{r}}_{\omega}^{G, u p}(t)=\dot{\mathbf{r}}_{\omega}^{G, u p} \mathbf{w}^{(Q-1)}(t), \\
& \dot{\mathbf{r}}_{\omega}^{G, d n}(t)=\dot{\mathbf{r}}_{\omega}^{G, d n} \mathbf{w}^{(Q-1)}(t) \\
& \dot{\mathbf{r}}_{\omega}^{S, u p}(t)=\dot{\mathbf{r}}_{\omega}^{S, u p} \mathbf{w}^{(Q-1)}(t), \\
& \dot{\mathbf{r}}_{\omega}^{S, d n}(t)=\dot{\mathbf{r}}_{\omega}^{S, d n} \mathbf{w}^{(Q-1)}(t),
\end{aligned}
$$

The renewable energy spillage trajectory is spanned as:

$$
S P_{\omega}(t)=\mathbf{S P}_{\omega} \mathbf{w}^{Q}(t) .
$$

The proposed function space solution model projects the first stage and second stage decision trajectories into the Bernstein function space in (49)-(67). Using the models proposed in our previous works $[17,18]$, it is 
straightforward to show that the objective functional and the continuous-time equality constraints of the proposed model (1)-(46) can be converted into algebraic equations on the Bernstein coefficients of the decisions trajectories. In addition, the convex hull property of the Bernstein polynomials can be utilized to convert the continuous-time inequality constraints into inequality constraints on the the Bernstein coefficients of the decisions trajectories $[17,18]$. We refer the readers to [17-19] for more information about the function space solution model.

\section{Numerical Studies}

The proposed model is implemented on the IEEE-RTS [20]. The 5-min historical real-time load and solar power data of CAISO are utilized for the studies [21]. The data from three consecutive three consecutive Mondays from Nov. 5 to Nov. 19, 2018 are utilized to construct a Gaussian process model for load and solar power generation, and after applying scenario reduction process, twenty load and solar power scenarios are utilized for computational efficiency. More details about constructing the Gaussian process models and generating scenarios is provided in [18, 22].

The generation cost, start-up, and shut-down costs of the generating units are taken from [20]. The up and down flexibility reserve capacity cost, flexibility deployment cost, as well as flexible ramping cost of generating units are respectively $40 \%, 140 \%$, and $10 \%$ of the highest piecewise linear generation cost. An ES system with energy capacity, power rating, and ramping rate of respectively $1200 \mathrm{MWh}, 250 \mathrm{MW}$, and $25 \mathrm{MW} / \mathrm{min}$ is added to the test system. The ES charging and discharging efficiency coefficients are both $90 \%$. The ES system submits two charging and discharging bids, where the charging and discharging bids for $0 \leq$ $G^{s}(t), D^{s}(t) \leq 125$ are respectively $\$ 10.5 / \mathrm{MW}$ and $\$ 18.5 / \mathrm{MW}$ and the bids for $125<G^{s}(t), D^{s}(t) \leq 250$ are respectively $\$ 10 / \mathrm{MW}$ and $\$ 19 / \mathrm{MW}[18]$. The up and down flexibility reserve capacity costs of ES are both $\$ 5 / \mathrm{MWh}$. The up and down flexibility deployment costs of ES are both $\$ 13 / \mathrm{MWh}$, while the flexible ramping cost is $\$ 2$ per $\mathrm{MW} / \mathrm{h}$. The solar power spillage cost is \$100/MW.

The operation of the test system is simulated using the proposed model for a 24-hour day-ahead scheduling horizon with an optimality gap of $0.2 \%$, given different values of risk aversion parameter $\beta$, and the confidence level $\alpha$ for risk management is considered to be $90 \%$. The load and solar scenarios as well as the decision trajectories are modeled by Bernstein polynomials of degree 3. The expected total operation costs and CVaR values of the system under different risk aversion levels

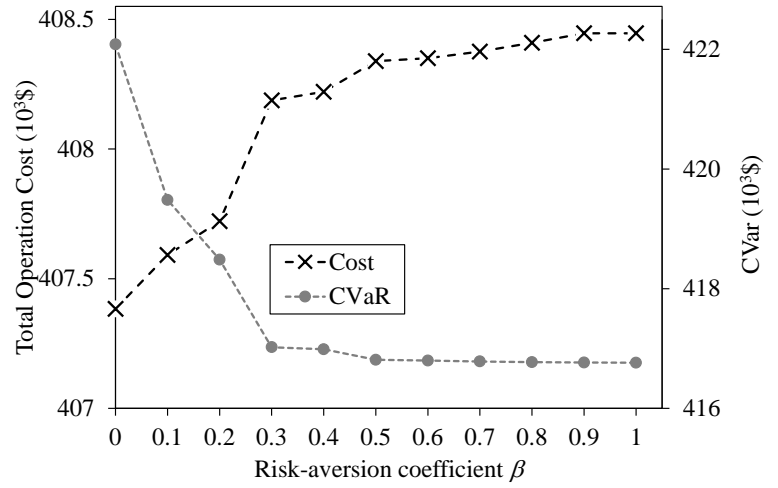

Figure 2. Operation cost and CVaR for different values of $\beta$

are illustrated in Fig. 2 (note that the first and last data points on each curve in Fig. 2 are respectively associated with $\beta=0.01$ and $\beta=0.99$ ). In Fig. 2, the higher the value of risk aversion coefficient $\beta$ is, the more risk-averse the system operator is. Hence, the expected total system operation cost increases with the increase in $\beta$ due to the increased amount of the of costs associated with providing reserve capacity. However, CVaR, representing the average of the $10 \%$ higher tail of the scenario costs in ascending order, decreases with the increase of $\beta$.

The total scheduled flexibility reserve capacity trajectories under different risk aversion levels are shown in Fig. 3. In Fig. 3, the total up flexibility reserve capacity is the same under different risk aversion levels since the system has to reserve sufficient capacity considering the upward net-load variation for all uncertain scenarios. However, as the value of $\beta$ increases, the model schedules more down flexibility reserve to avoid the underlying uncertainty from solar penetration, as shown in Fig. 4. The ES flexibility reserve capacity trajectories under different risk aversion levels are illustrated in Fig. 5. In Fig. 5, the lower the

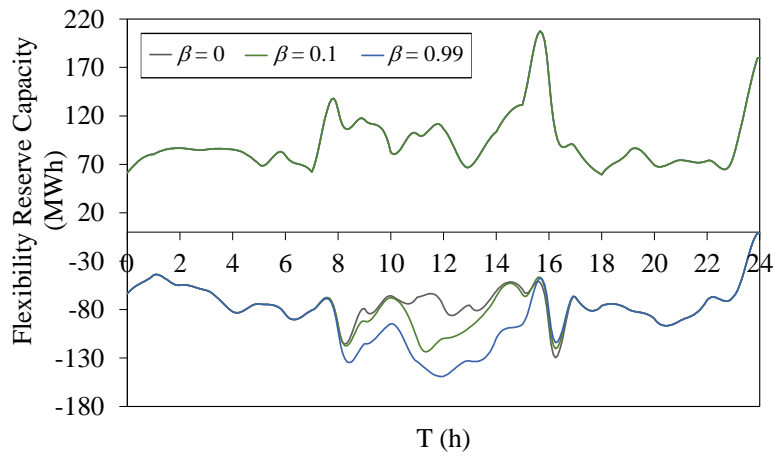

Figure 3. Total flexibility reserve capacity under different risk aversion levels 

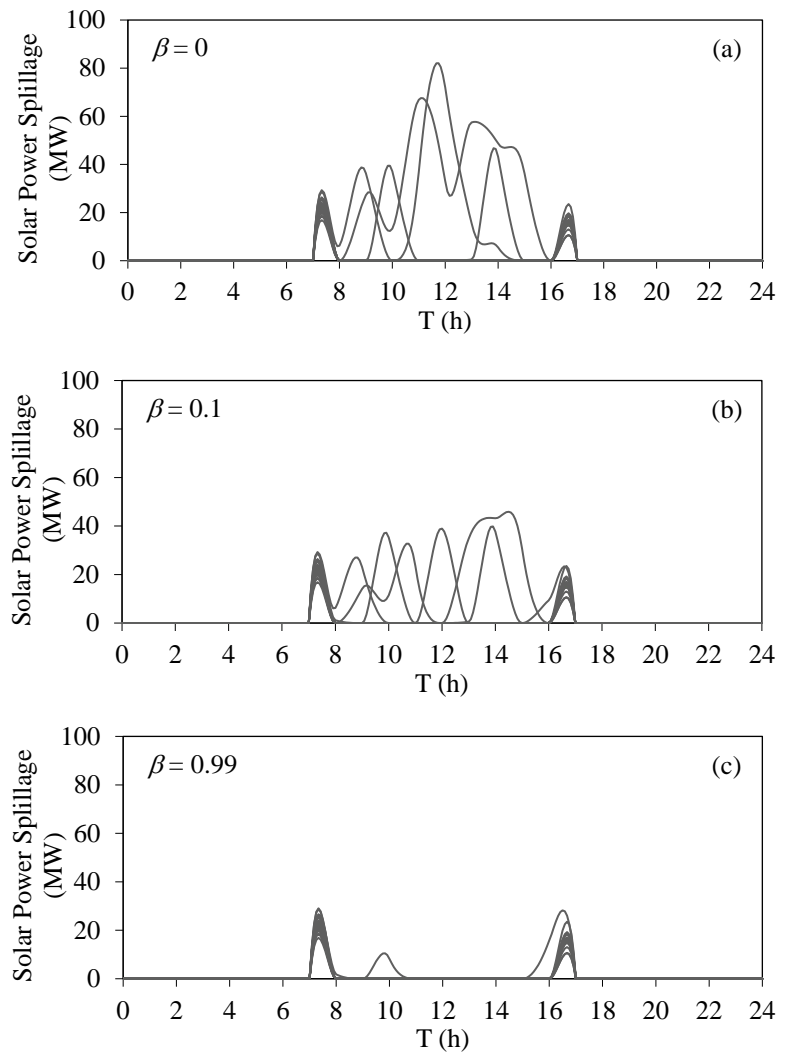

Figure 4. Solar power spillage trajectories in differnet scenarios (a) $\beta=0$, (b) $\beta=0.1$, (c) $\beta=0.99$

value of $\beta$ is, the less ES is utilized for providing down flexibility reserve. This is because as $\beta$ decreases, the system becomes less risk-averse, and solar generation is spilled more rather than employing flexibility for utilizing more uncertain solar power in the system.

The total amounts of flexibility reserve capacity employed from the ES system and generating units in a day under different risk aversion levels is depicted in Fig. 6. In Fig. 6, under different risk aversion levels, the system can choose between reserve capacity for down flexibility reserve to deal with the uncertainty or to use solar spillage to avoid the underlying uncertainty. Thus, with the increase in $\beta$, less solar spillage is and more total down flexibility reserve is deployed, which is consistent with Fig. 3 and Fig. 4. However, for the up flexibility reserve, the system has to reserve enough capacity to compensate for the uncertainty in each scenario. Thus, the total up flexibility reserve capacity remains the same under different $\beta$ values.

The expected total operation costs and $\mathrm{CVaR}$ values under different uncertainty levels, as represented by the number of scenarios in the model, are presented for $\beta=$ 0.1 in Table 1. In Table 1, as more uncertain scenarios are employed in the proposed model, the expected

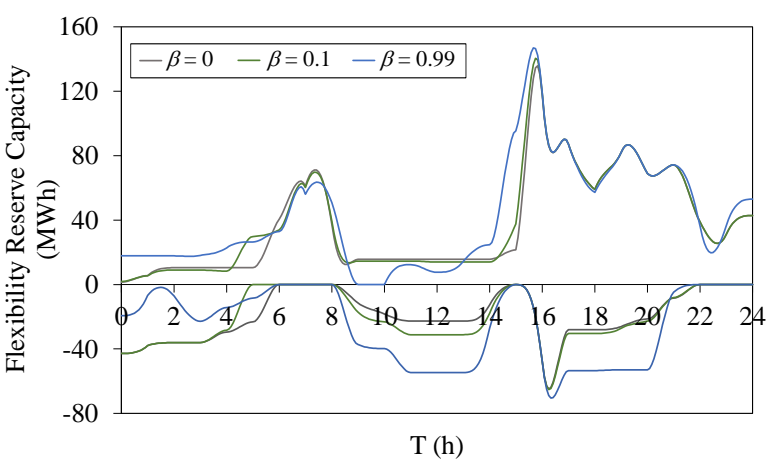

Figure 5. ES flexibility reserve capacity trajectories

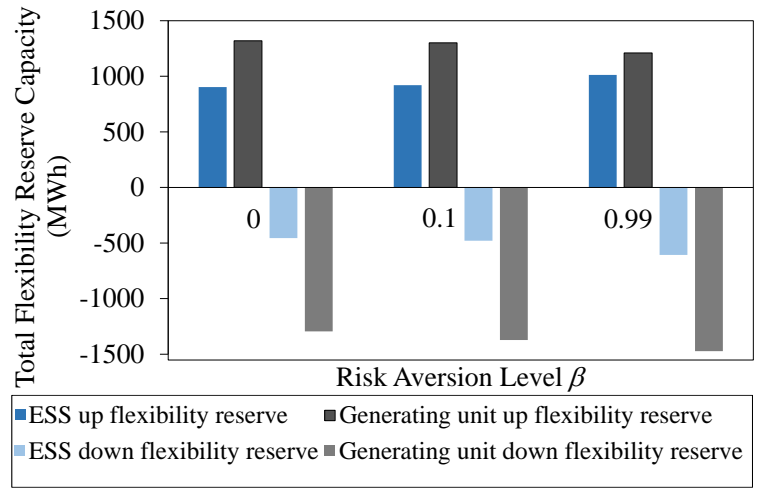

Figure 6. Total flexibility reserve capacity of the ES system and generating units

total operation cost and CVaR both increase due to the higher level of uncertainty taken into consideration by the model. The ES system contributes more ramping capability to the system as a higher level of uncertainties are taken into consideration, shown in Fig. 7. In Fig. 7 , as the number of uncertain scenarios increases, the ES provides more ramping capability in each scenario in addition to the ramping trajectory determined by the charging/discharging power in the first-stage.

Table 1. Cost and CVaR Analysis

\begin{tabular}{cccc}
\hline Number of Scenarios & 15 & 20 & 35 \\
\hline Expected Cost (\$) & 404,363 & 407,591 & 412,281 \\
CVaR (\$) & 417,249 & 419,482 & 426,031 \\
\hline
\end{tabular}

\section{Conclusion}

This paper proposed a risk-based stochastic continuous-time flexibility reserve scheduling model for optimizing the contribution of ES flexibility to compensate the uncertainty of renewable generation at a given risk tolerance level. CVaR is employed 

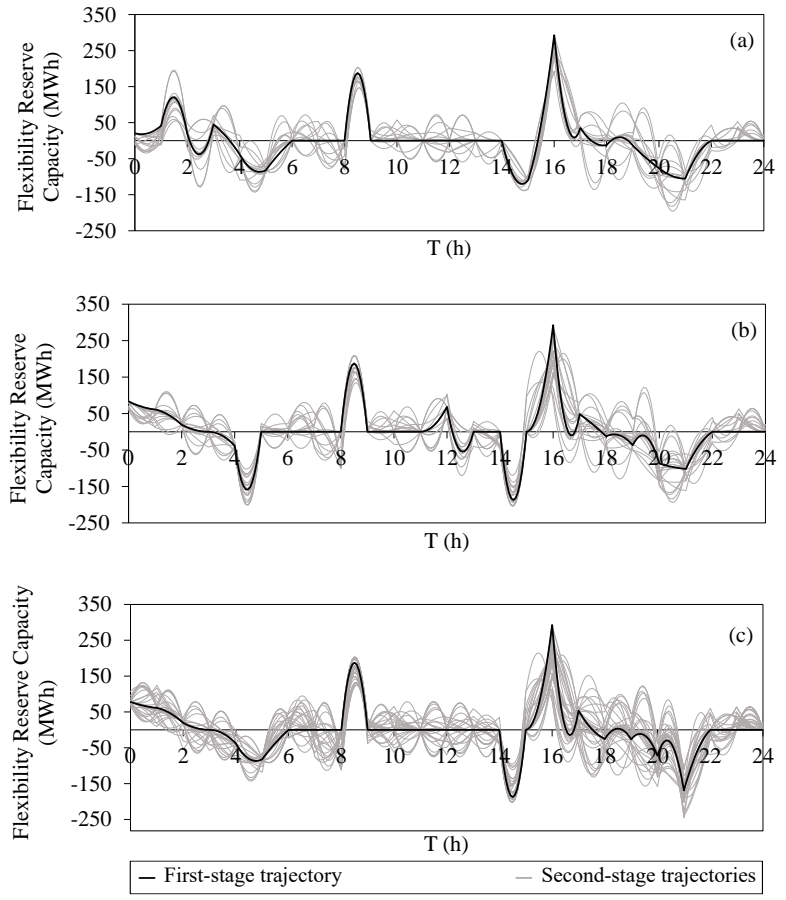

Figure 7. ES ramping trajectories (a) 15 scenarios (b) 20 scenarios (c) 35 scenarios

as the financial risk metric of the system operation, measuring the financial risk imposed by the uncertainty of load and renewable generation. The proposed continuous-time optimization problem is solved using a function space solution method that converts the problem into a scalable MILP problem. The proposed model is implemented on IEEE-RTS using load and solar power data from CAISO. Numerical results show that ES system as well as generating units contribute to managing the uncertainty of load and RES generation, through providing flexibility reserve and ramping capability to the system. The results indicate that when the system operator is more risk-averse, more flexibility capacity is deployed from the ES system and generating units, supporting the integration of uncertain renewable generation rather than curtailing it.

\section{Acknowledgement}

This research was funded by the U.S. Department of Energy Office of Electricity Energy Storage program under the guidance of Dr. Imre Gyuk. Sandia National Laboratories is a multimission laboratory managed and operated by National Technology Engineering Solutions of Sandia, LLC, a wholly owned subsidiary of Honeywell International Inc., for the U.S. Department of Energy's National Nuclear Security Administration under contract DE-NA0003525.

\section{References}

[1] K. Oikonomou, V. Satyal, and M. Parvania, "Energy storage in the western interconnection: Current adoption, trends and modeling challenges," in 2017 IEEE PES General Meeting, pp. 1-5, 2017.

[2] "Order no. 841: Electric storage participation in markets operated by regional transmission organizations and independent system operators," 2018.

[3] N. Padmanabhan, M. Ahmed, and K. Bhattacharya, "Battery energy storage systems in energy and reserve markets," IEEE Transactions on Power Systems, vol. 35, no. 1, pp. 215-226, 2019.

[4] R. Khatami, K. Oikonomou, and M. Parvania, "Look-ahead optimal participation of compressed air energy storage in day-ahead and real-time markets," IEEE Transactions on Sustainable Energy, vol. 11, no. 2, pp. 682-692, 2020.

[5] J. Hu, M. R. Sarker, J. Wang, F. Wen, and W. Liu, "Provision of flexible ramping product by battery energy storage in day-ahead energy and reserve markets," IET Generation, Transmission \& Distribution, vol. 12, no. 10, pp. 2256-2264, 2018.

[6] T. A. Nguyen, R. H. Byrne, R. J. Concepcion, and I. Gyuk, "Maximizing revenue from electrical energy storage in miso energy \& frequency regulation markets," in 2017 IEEE PES General Meeting, pp. 1-5, 2017.

[7] T. A. Nguyen, D. A. Copp, and R. H. Byrne, "Stacking revenue from energy storage providing resilience, t\&d deferral and arbitrage," in 2019 IEEE PES General Meeting, pp. 1-5, 2019.

[8] P. Zou, Q. Chen, Q. Xia, G. He, and C. Kang, "Evaluating the contribution of energy storages to support large-scale renewable generation in joint energy and ancillary service markets," IEEE Transactions on Sustainable Energy, vol. 7, no. 2, pp. 808-818, 2015.

[9] R. Khatami, M. Parvania, and P. Khargonekar, "Continuous-time look-ahead scheduling of energy storage in regulation markets," in Proceedings of the 52nd Hawaii international conference on system sciences, 2019.

[10] B. Li, A. Bagherinezhad, R. Khatami, and M. Parvania, "Continuous-time look-ahead optimization of energy, storage in real-time balancing and regulation markets," IEEE Systems Journal, 2020.

[11] T. Rodrigues, P. J. Ramírez, and G. Strbac, "Risk-averse bidding of energy and spinning reserve by wind farms with on-site energy storage," IET Renewable Power Generation, vol. 12, no. 2, pp. 165-173, 2017.

[12] P. Aliasghari, M. Zamani-Gargari, and B. Mohammadi-Ivatloo, "Look-ahead risk-constrained scheduling of wind power integrated system with compressed air energy storage (caes) plant," Energy, vol. 160, pp. 668-677, 2018.

[13] M. Jadidbonab, E. Babaei, and B. Mohammadi-ivatloo, "Cvar-constrained scheduling strategy for smart multi carrier energy hub considering demand response and compressed air energy storage," Energy, vol. 174, pp. 1238-1250, 2019.

[14] M. Jadidbonab, A. Dolatabadi, B. Mohammadi-Ivatloo, M. Abapour, and S. Asadi, "Risk-constrained energy management of pv integrated smart energy hub in the presence of demand response program and compressed air energy storage," IET Renewable Power Generation, vol. 13, no. 6, pp. 998-1008, 2019. 
[15] Y. Zhang, X. Han, B. Xu, M. Wang, P. Ye, and Y. Pei, "Risk-based admissibility analysis of wind power integration into power system with energy storage system," IEEE Access, vol. 6, pp. 57400-57413, 2018.

[16] H. Wu, M. Shahidehpour, A. Alabdulwahab, and A. Abusorrah, "A game theoretic approach to risk-based optimal bidding strategies for electric vehicle aggregators in electricity markets with variable wind energy resources," IEEE Transactions on Sustainable Energy, vol. 7, no. 1, pp. 374-385, 2015.

[17] M. Parvania and A. Scaglione, "Unit commitment with continuous-time generation and ramping trajectory models," IEEE Transactions on Power Systems, vol. 31, no. 4, pp. 3169-3178, 2016.

[18] R. Khatami, M. Parvania, and A. Narayan, "Flexibility reserve in power systems: Definition and stochastic multi-fidelity optimization," IEEE Transactions on Smart Grid, vol. 11, no. 1, pp. 644-654, 2020.

[19] R. Khatami and M. Parvania, "Stochastic multi-fidelity scheduling of flexibility reserve for energy storage," IEEE Transactions on Sustainable Energy, vol. 11, no. 3, pp. 1438-1450, 2020.

[20] C. Grigg, P. Wong, P. Albrecht, R. Allan, M. Bhavaraju, R. Billinton, Q. Chen, C. Fong, S. Haddad, S. Kuruganty, et al., "The ieee reliability test system-1996. a report prepared by the reliability test system task force of the application of probability methods subcommittee," IEEE Transactions on power systems, vol. 14, no. 3, pp. 1010-1020, 1999.

[21] California ISO Open Access Same-Time Information System. [Online]. Available: http://oasis.caiso.com.

[22] R. Khatami, M. Parvania, P. Khargonekar, and A. Narayan, "Continuous-time stochastic modeling and estimation of electricity load," in 2018 IEEE Conference on Decision and Control (CDC), pp. 3988-3993, 2018. 